\title{
Estudo epidemiológico de urgências odontológicas da FOP/UPE
}

\section{Epidemiological study of dental emergencies of the FOP/UPE}

\author{
Elivalter Pereira Martins* \\ Osmiely Reis de Oliveira** \\ Silvia Regina Sampaio Bezerra** \\ Adriane Tenório Dourado ${ }^{* * *}$
}

\section{Resumo}

Objetivo: este estudo avalia a prevalência das urgências odontológicas, bem como os tratamentos mais frequentes realizados no serviço de urgência da Faculdade de Odontologia da Universidade de Pernambuco (FOP/ UPE). Materiais e método: trata-se de um estudo retrospectivo, de base de dados secundários, utilizando como instrumento de pesquisa os prontuários dos atendimentos de urgência compreendidos no período de 2007 a 2011. Os dados foram coletados por meio de um formulário padrão. O nível de significância, considerado nos testes estatísticos, foi de 5\%, e foram utilizadas técnicas estatísticas descritivas, por meio da elaboração de frequências absolutas e percentuais, e das medidas de estatística: média, desvio-padrão e mediana. O programa estatístico usado para análise dos dados foi o Statistical Package for the Social Sciences (SPSS) versão 17. Resultados: foram obtidos 862 prontuários, com pacientes de ambos os sexos, sendo o feminino predominante (70\%), com idade dos pacientes variando entre 12 anos e 81 anos, e a queixa mais frequente a dor de origem dental $(80,3 \%)$. O diagnóstico mais prevalente foi a pulpite irreversível sintomática $(29,6 \%)$, seguido pelo abcesso dento-alveolar agudo $(7,8 \%)$, bem como a pulpectomia $(26,8 \%)$ como o tratamento de urgência mais realizado no serviço odontológico da instituição. Conclusões: pode-se inferir que a dor foi o principal motivo da procura dos pacientes no serviço de urgência odontológica da FOP/UPE, refletindo o alto percentual das urgências endodônticas no âmbito odontológico. Portanto, este trabalho contribui para o desenvolvimento da investigação epidemiológica das urgências odontológicas no sentido amplo do contexto de saúde bucal da população.

Palavras-chave: Epidemiologia. Urgência. Dor.

\section{Introdução}

Os termos urgência e emergência são aplicados em muitas ocasiões, no âmbito dos serviços de saúde, como se apresentassem significados semelhantes. Um dos riscos possíveis, advindos de tal fato, é a banalização dos termos, de tal forma que sejam usados sem que se saiba efetivamente o que são ${ }^{1}$. No glossário odontológico de Simas $^{2}$ (1989), esses termos são distintos para o autor, ou seja, emergência é todo incidente ou ocorrência que causa ameaça não somente à boa marcha da operação como à saúde e à vida do paciente. Como os serviços de pronto atendimento odontológicos não englobam situações de risco iminente de vida, o termo urgência odontológica é de uso mais apropriado ${ }^{3}$.

A precária condição de saúde bucal dos brasileiros é um reflexo da grande desigualdade socioeconômica vigente e do difícil acesso à assistência odontológica, decorrente da oferta limitada dos serviços públicos e do alto custo que a prática privada representa para grande parte da população. Enquanto os indivíduos da classe média e alta têm acesso a todos os recursos tecnológicos disponíveis para tratamento curativo e preventivo, procurando pelos serviços odontológicos para a manutenção de sua saúde bucal, as pessoas de classe baixa recorrem ao profissional principalmente em casos de dor $^{4-6}$. Em muitos casos, os serviços odontológicos

Cirurgião-dentista pela Faculdade de Odontologia de Pernambuco na Universidade de Pernambuco (UPE), Camaragibe, PE, Brasil.

Cirurgiã-dentista pela Faculdade de Odontologia de Pernambuco na Universidade de Pernambuco (UPE), Camaragibe, PE, Brasil.

Doutora em Dentística/Endodontia; professora adjunta da Clínica de Atenção Básica III da Faculdade de Odontologia de Pernambuco na Universidade de Pernambuco (UPE), Camaragibe, PE, Brasil.

**** Doutora em Endodontia; professora adjunta da Clínica de Atenção Básica III da Faculdade de Odontologia de Pernambuco na Universidade de Pernambuco (UPE), Camaragibe, PE, Brasil. 
de urgência, oferecidos de forma gratuita por órgãos públicos ou instituições de ensino, são a única solução para a resolução dos problemas de dor de origem bucal da população desfavorecida socioeconomicamente ${ }^{7-8}$.

Vários estudos apontam que, mesmo em locais com uma rede e um modelo de atenção à saúde organizada, hierarquizada a partir da atenção básica, os casos de urgência odontológica são frequentes, necessitando de cuidados específicos, para os quais os centros de referência podem ser uma solução para aumentar a resolutividade do modelo de atenção proposto ${ }^{9-11}$. Nesse contexto, sendo o atendimento de urgência prioritário, o paciente que não consegue acessar o serviço para seu tratamento de rotina acaba por buscar esse atendimento, seja por apresentar um quadro agudo, com presença de dor, ou por necessitar da realização de um procedimento que, para o paciente, é urgente, ainda que não se configure como tal, do ponto de vista estritamente biológico ${ }^{1}$.

O principal motivo para os pacientes procurarem o atendimento odontológico nas faculdades de odontologia é, normalmente, a situação de urgência, em que a dor é sempre presente ${ }^{7,12-13}$. Para alguns autores, a maioria dos casos de urgência e dor de origem odontológica é causada por problemas endodônticos, dentre os quais o mais comum é a pulpite irreversível sintomática ${ }^{12}$.

A urgência odontológica faz parte da atenção básica à saúde, sendo uma ocorrência de rotina na vida profissional dos cirurgiões-dentistas ${ }^{14}$. Por meio de estudos epidemiológicos, subsídios são fornecidos para estimar as condições atuais de saúde bucal e as necessidades de tratamento da população, auxiliando no monitoramento das alterações nos níveis e padrões das doenças. É imprescindível, portanto, que o cirurgião-dentista esteja familiarizado com esses aspectos epidemiológicos, buscando nesses a base para a prevenção, para o diagnóstico e para o tratamento das alterações bucais ${ }^{15}$.

Assim, por ser a urgência uma realidade vivida no cotidiano da clínica odontológica, o propósito desta pesquisa foi avaliar a prevalência dessas necessidades odontológicas atendidas na Faculdade de Odontologia da Universidade de Pernambuco (FOP/UPE)

\section{Materiais e método}

Foi realizado um estudo retrospectivo, quantitativo, transversal na Faculdade de Odontologia de Pernambuco, localizada na cidade de Camaragibe, região Metropolitana do Recife, Pernambuco, no período de fevereiro a julho de 2012.

A população estudada foi constituída pelas fichas clínicas dos pacientes atendidos nas urgências odontológicas da Clínica Integrada da Faculdade de
Odontologia de Pernambuco. A amostra deste estudo retrospectivo foi constituída por 862 prontuários de pacientes de ambos os sexos, com idade a partir de 12 anos, atendidos no serviço de urgência da FOP/UPE, no período entre 2007 e 2011. A partir desse período, foi verificada a seleção da amostra, utilizando as fichas clínicas de urgência, preenchidas por alunos do nono período de graduação desse centro universitário, sob supervisão e orientação dos professores da disciplina da Clínica Integrada, que é responsável pelo serviço de urgência dessa instituição de ensino superior.

Antes da seleção da amostra, utilizou-se como critérios de exclusão as fichas clínicas que não pertenciam ao setor de atendimento da urgência, bem como aquelas que não se encontravam totalmente preenchidas com os dados sociodemográficos relativos ao paciente, em razão da procura ao atendimento de urgência e/ou diagnóstico, ou tratamento realizados pelos acadêmicos e as fichas fora do período estabelecido para a realização da pesquisa científica. Dessa forma, não ocorreram perdas na coleta de dados das fichas pesquisadas.

$\mathrm{O}$ instrumento de pesquisa utilizado para a coleta de dados foi um formulário padrão para o preenchimento dos dados obtidos no levantamento epidemiológico, com base de dados secundários. Nesse formulário, foram anotados dados dos prontuários referentes ao paciente para arquivamento e totalização dos dados e das variáveis, permitindo uma maior fidedignidade e veracidade das informações, evitando-se, dessa forma, falha de memória e/ou distorções das informações coletadas. As variáveis relativas às condições de cada paciente, encontradas nos prontuários, importantes para compreender como o processo saúde-doença bucal é percebido no nível epidemiológico, foram incluídas no formulário aplicado no estudo. As variáveis utilizadas definiram os dados contidos nesses prontuários (idade, gênero, queixa principal expressa pelo paciente, diagnóstico e tratamento indicado).

$\mathrm{Na}$ análise estatística, os dados do formulário foram avaliados e digitados em um banco de dados e importados para o programa estatístico Statistical Package for the Social Sciences (SPSS) da versão 17.0. Os dados foram analisados por intermédio das técnicas de estatística descritiva, por meio da elaboração de frequências absolutas e percentuais e das medidas de estatística - média, desvio-padrão e mediana -, sendo previamente estabelecida a margem de erro padrão de $5 \%$ e um intervalo de confiança de $95 \%$ ( $\mathrm{IC}=95 \%$ ).

De acordo com a resolução 196/96 do Conselho Nacional de Saúde, justifica-se a não apresentação do Termo de Consentimento Livre e Esclarecido, uma vez que a presente pesquisa usou dados secundários, não tendo, portanto, contato com humanos. Foi solicitada a anuência 
da direção da Faculdade de Odontologia de Pernambuco para o início da pesquisa, bem como da disciplina responsável pelo setor de urgência. Este estudo foi encaminhado ao Comitê de Ética em Pesquisa da Universidade de Pernambuco, pelo qual foi aprovado sob registro CAAE: 01360097000-11.

\section{Resultados}

Foram analisados 862 prontuários do estudo, em que a idade dos pesquisados variou de 12 a 81 anos, teve média de 33,04 anos, mediana de 31 anos e desvio-padrão de 13,36 anos. Na Tabela 1, apresentam-se os resultados relativos ao perfil sociodemográfico dos pacientes.

Na Figura 1, evidenciam-se os resultados relativos às queixas principais dos pacientes pesquisados no estudo.

Pode-se analisar nas Figuras 2 e 3 as variáveis (diagnóstico e tratamento) mais prevalentes na urgência, assim como a presença dos outros diagnósticos e tratamentos que não são urgências propriamente ditas, as quais são ilustradas na Tabela 2, a seguir.
Tabela 1 - Distribuição dos pesquisados, segundo os dados sociodemográficos. Camaragibe-PE, 2012

\begin{tabular}{l|r|r}
\hline \multicolumn{1}{c|}{ Variável } & $\mathrm{n}$ & $\%$ \\
\hline Total & 862 & 100 \\
Faixa etária (anos) & 146 & 16,9 \\
12 a 19 & 246 & 28,5 \\
20 a 29 & 221 & 25,6 \\
30 a 39 & 145 & 16,8 \\
40 a 49 & 67 & 7,8 \\
50 a 59 & 37 & 4,3 \\
60 anos ou mais & & \\
Gênero & 259 & 30 \\
Masculino & 603 & 70 \\
Feminino & & \\
\hline Fonte: dos autores.
\end{tabular}

Fonte: dos autores.

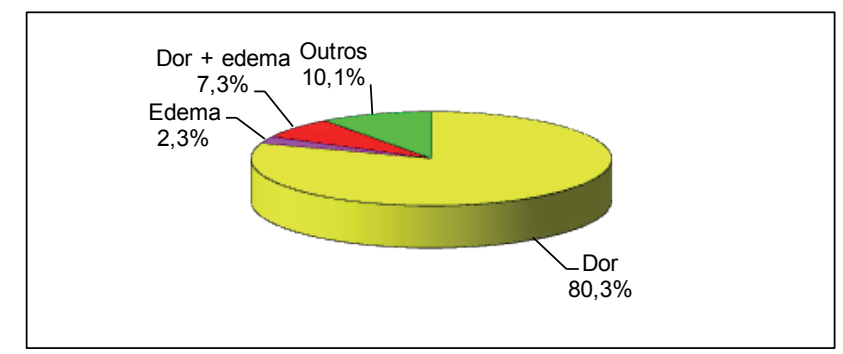

Fonte: dos autores.

Figura 1 - Distribuição dos pesquisados, segundo a razão da queixa principal. Camaragibe-PE, 2012

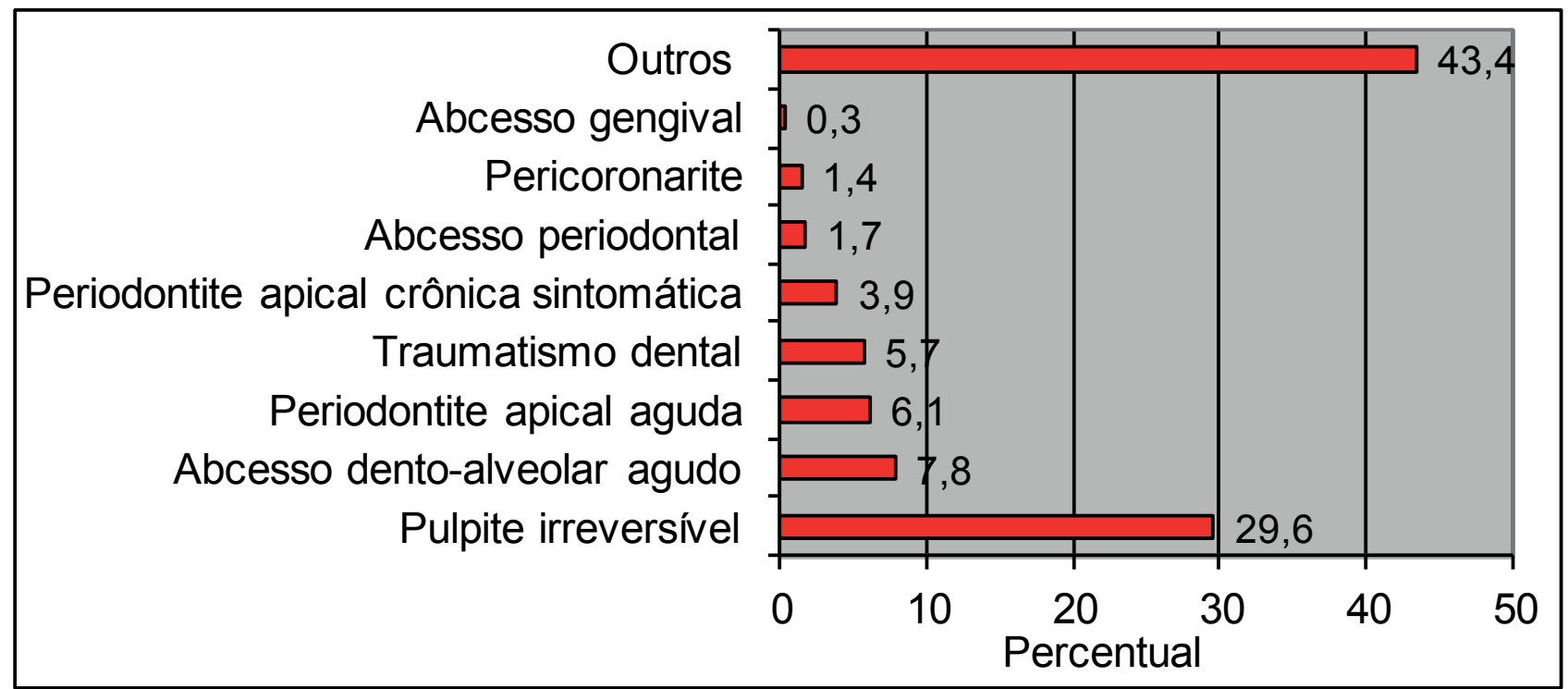

Fonte: dos autores.

Figura 2 - Distribuição dos pesquisados segundo os diagnósticos mais presentes. Camaragibe-PE, 2012 


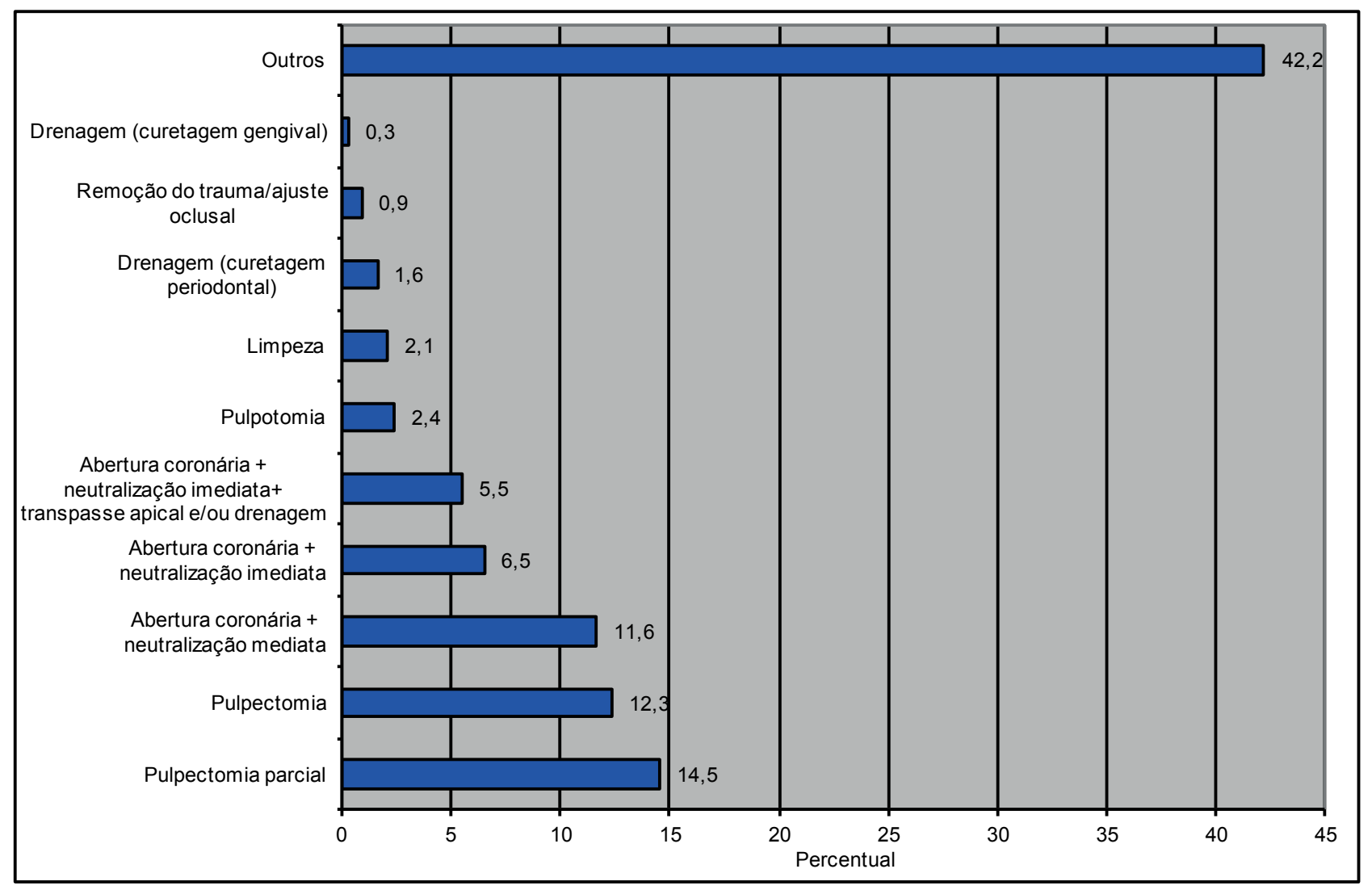

Fonte: dos autores.

Figura 3 - Distribuição dos pesquisados, segundo os tratamentos mais presentes. Camaragibe-PE, 2012

Tabela 2 - Distribuição dos pesquisados segundo os outros diagnósticos e tratamentos não categorizados como urgências. Camaragibe-PE, 2012

\begin{tabular}{l|r|r}
\hline \multicolumn{1}{c|}{ Variável } & $\mathrm{n}$ & \multicolumn{1}{c}{$\%$} \\
\hline Total & & \\
Outros diagnósticos & 374 & 43,4 \\
- Pultite reversível & 103 & 11,9 \\
- Necrose pulpar & 64 & 7,6 \\
- Cárie dentária & 30 & 3,5 \\
- Resto radicular & 11 & 1,3 \\
- Fratura de restauração & 27 & 3,1 \\
- Abcesso dento-alveolar crônico & 13 & 1,5 \\
- Doença periodontal & 25 & 2,9 \\
- Sensibilidade dentinária & 26 & 3,0 \\
- Periodontite apical crônica & 15 & 1,7 \\
- Alveolite & 03 & 0,3 \\
- Outros problemas não especificados & 57 & 6,6 \\
Outros tratamentos & 374 & 43,4 \\
- Medicação sistêmica & 49 & 5,7 \\
- Capeamento pulpar indireto & 69 & 8,0 \\
- Capeamento pulpar direto & 33 & 3,8 \\
- Exodontia & 24 & 2,8 \\
- Restauração definitiva & 92 & 10,7 \\
- Encaminhamento & 59 & 6,8 \\
- Outros tratamentos não especificados & 48 & 5,6 \\
\hline Fonte dos autors.
\end{tabular}

Fonte: dos autores.

\section{Discussão}

É notável que exista uma alta necessidade de tratamento principalmente devido à cárie dentária e suas sequelas, bem como sua associação a questões sociais. Já que as pessoas menos favorecidas tem menos acesso aos serviços de atenção à saúde bucal, levando à procura excessiva pelos serviços de urgência odontológica especialmente nas instituições de ensino. Nesse sentido, o paciente busca a partir do atendimento de urgência uma porta de entrada para ver solucionado o seu problema de saúde bucal, mesmo que não se enquadre nos padrões conceituais da urgência ${ }^{12}$. Desse modo, é importante que as urgências odontológicas sejam tratadas como prioridade dentro do planejamento de tratamentos odontológicos integrados ${ }^{16}$.

De acordo com os resultados obtidos, a maioria dos atendimentos de urgência foi realizada em pacientes do gênero feminino. Esses dados são compatíveis com outros estudos semelhantes ${ }^{1,3,11,12,17-21}$, nos quais também foi evidenciada maior ocorrência de mulheres na população investigada. Uma das prováveis justificativas encontradas para tal resultado é que no Brasil o percentual de mulheres é maior do que o de homens, segundo os dados divulgados pelo Instituto Brasileiro de Geografia e Estatística $(\mathrm{IBGE})^{22}$. Para a literatura, essa diferença é plausível, uma vez que a procura pelos serviços odontológi- 
cos é maior por parte das mulheres em comparação aos homens, já que concebem maior importância à estética e à saúde como serviço regular em sua rotina ${ }^{3}$. Já a presença masculina é mais prevalente do que a feminina em serviços de emergência odontológica, devido aos acidentes automobilísticos ou aos acidentes resultantes de violências ${ }^{19,23}$. No entanto, alguns autores encontraram resultados diferentes em seus estudos, nos quais comprovaram que, no gênero masculino, as urgências são mais frequentes que no feminino $^{24}$. Provavelmente, isso é devido à menor procura por atendimento pelo gênero masculino em relação ao feminino, no campo da saúde, para o tratamento ambulatorial, gerando um afluxo maior por parte dos homens nos serviços de tratamento tardio.

Entretanto, alguns pesquisadores concordam que o principal motivo para a procura por um atendimento de urgência seja a dor, independente do gênero ${ }^{3,10}$, tal fato é corroborado no presente estudo, uma vez que a dor foi a principal queixa do paciente. Por esse motivo, quanto maior a procura pelo serviço, maior o número de dentes restaurados e perdidos. Consequentemente, há diante disso, uma possibilidade maior de sequelas aos dentes, quando negligenciados por parte do profissional ou mesmo do paciente.

Foi observado, neste estudo, que a faixa etária que mais utilizou o serviço de urgência estava entre 20 e 29 anos, fato confirmado com outros estudos ${ }^{1,7,12,16}$. Por ser uma doença que acomete mais crianças e adultos jovens, a diminuição da incidência de cárie dentária é a principal razão pela menor procura do serviço por pacientes com idades mais elevadas. Os resultados do projeto "SB Brasil 2010" ressaltam a importância do declínio da cárie dentária mais marcante em adultos com idade entre 35 e $44 \operatorname{anos}^{25}$.

Observa-se ainda um menor número de atendimento na idade odontopediátrica, o que deve-se, provavelmente, ao fato das crianças dependerem da percepção de seus pais a respeito das suas necessidades e de muitos relutarem em levar seus filhos ao dentista, além de existir a disciplina de Odontopediatria, na qual são realizados atendimentos de urgência, de forma restrita, cuidando, no setor de urgência da faculdade, da faixa etária dos maiores de 12 anos de idade ${ }^{3,26}$. Com base nos dados deste trabalho, verificou-se que o principal motivo da busca por atendimento no serviço de urgência foi o de dor (80,3\%), concordando com outros estudos de mesmo cunho científico ${ }^{3,17,19,27-28}$. Dentre as razões da dor odontológica, alterações relacionadas à cárie dentária respondem por aproximadamente $30 \%$ da prevalência desse tipo de dor ${ }^{29}$. Alguns artigos observaram que o grau de escolaridade dos indivíduos, a gravidade da cárie dentária, expressa por perdas dentais e o acesso a serviços odontológicos são fatores relacionados com dor de origem dental ${ }^{30-31}$, ou seja, a dor, além de interferir na qualidade de vida das pessoas, é influenciada pelas condições sociais e de acesso a serviços odontológicos.

$\mathrm{Na}$ presente pesquisa, a cárie dentária e suas consequências também foram significantes, dos diagnósticos relacionados à pulpite irreversível sintomática foi a mais prevalente, e esses achados são semelhantes a alguns artigos ${ }^{12,18}$ em que também foi mais prevalente essa patologia pulpar. A dor de origem pulpar ou periapical é um dos mais importantes problemas dentro de um serviço de urgência odontológica ${ }^{28}$. Entretanto, alguns autores realizaram um estudo em que $49 \%$ dos diagnósticos realizados pelos dentistas foram de doença de polpa e $13 \%$ de cárie dentária profunda ${ }^{21}$. Outros estudos encontraram como causa mais frequente de atendimento a pulpite, o abscesso periapical agudo, a cárie dentária profunda, a necrose pulpar, o abscesso periodontal e a fratura dental ${ }^{32}$. A explicação para essa grande frequência de outros diagnósticos inseridos em um setor de urgência de ensino superior pode ser devido à necessidade da busca por um atendimento odontológico, uma vez que o paciente procura a instituição como forma de suprir sua necessidade de tratamento no campo da saúde bucal, pois a saúde pública encontra-se defasada.

Neste trabalho, observou-se que a pulpectomia parcial foi o tratamento de urgência mais frequente, sendo seguido pelo pulpectomia com 12,3\%, considerados como a "primeira sessão canal" de dentes que necessitam de tratamento endodôntico radical. A causa provável para tal resultado pode ser em decorrência do tratamento ter uma forte relação com os diagnósticos mais prevalentes das urgências do serviço em questão, que são as patologias pulpares, principalmente as pulpites irreversíveis sintomáticas. Então, essa frequência significativa dos procedimentos relacionados à endodontia confirma que os profissionais responsáveis evitam, de alguma forma, que o paciente perca seus dentes, mesmo sabendo que, ao longo do tempo, o paciente talvez não se preocupe em salvá-los. Tais resultados são semelhantes a outros estudos, nos quais os tratamentos relacionados com a endodontia foram os mais realizados ${ }^{3,12}$.

Os resultados do estudo ora proposto divergem de alguns artigos como o de Sanchez e Drumond ${ }^{1}$ (2011), em que foi observado que o procedimento mais realizado no serviço odontológico de urgência da Clínica Integrada da Universidade Federal de Minas Gerais foi a exodontia, sendo os relacionados à endodontia o terceiro tratamento mais realizado. Contudo, é importante frisar que aqueles procedimentos que não foram conclusivos, ou seja, as pulpectomias e as aberturas coronárias, se não forem encaminhados a um lugar que se proponha a responder por aquela condição, serão responsáveis por novas urgências no futuro próximo, pois ao longo do tempo a condição bucal poderá se agravar.

A análise do perfil epidemiológico da população investigada demonstra um alto percentual correspondente aos outros diagnósticos, os quais não foram considerados urgências verdadeiras, sendo a mais frequente a pulpite reversível, seguida da necrose pulpar. Tal caracterização é explicada, provavelmente, devido à falta de acesso odontológico público de uma maneira equânime na população 
mais carente, resultando em uma maior procura ao serviço da faculdade para procedimentos que não são considerados como urgência. A literatura confirma que uma grande parcela da população depende, quase que exclusivamente, da prestação de serviços odontológicos públicos e, frente à sua escassez, é gerado um afluxo constante aos serviços de urgência ${ }^{8}$.

Os serviços de urgência odontológica parecem completar o modelo de hierarquização dos serviços de saúde bucal pública, conduzindo o usuário a buscar resolução em nível secundário para seus problemas de ordem ambulatorial básica ${ }^{11}$. Contudo, em consonância com o exposto anteriormente pelos autores, é importante lembrar que o serviço de urgência abordado por este estudo é alocado em uma instituição de ensino superior, e quando o paciente necessita de tratamento endodôntico, cirúrgico, clínico ou de outra complexidade, ele deve ser encaminhado para a disciplina específica. Entretanto, como o paciente chega ao serviço com queixa de dor (mesmo não apresentando), o acadêmico faz o procedimento que não é urgência, justificando, significativamente, o registro dos outros tratamentos, como por exemplo, os endodônticos e os restauradores definitivos, de nível ambulatorial.

É possível afirmar que os tratamentos decorrentes da cárie dentária e suas sequelas, isto é, a pulpectomia parcial, a pulpectomia, a abertura coronária mais a neutralização mediata ou imediata e/ou transpasse apical e drenagem, e a pulpotomia foram responsáveis por mais da metade dos procedimentos realizados na urgência da instituição pesquisada. Portanto, a cárie dentária e suas sequelas continuam sendo as principais causas das urgências odontológicas ${ }^{19}$, fato esse que corroborou com 0 presente estudo.

Diante do exposto, muitas faculdades de odontologia, aqui incluída a da Universidade de Pernambuco, têm clínicas que permitem aos seus acadêmicos atender uma parcela da população de um determinado local, sendo a ocorrência de urgências nessas clínicas um fato diário. A importância de se estudar as urgências em odontologia encontra-se no fato de que esses procedimentos são uma realidade vivida no cotidiano da clínica odontológica, já existindo alguns estudos interessantes a respeito desse tipo de atendimento, assim como ser propulsora de estratégias para qualificar o atendimento odontológico do serviço prestado à população, fazendo com que as urgências odontológicas, realizadas nas universidades, proporcionem bons resultados, tornando o serviço centro de referência para a população. Portanto, este estudo contribui para o desenvolvimento da investigação epidemiológica das urgências odontológicas, no sentido amplo do contexto de saúde bucal, da população atendida nos centros de urgências das instituições de ensino superior.

Pode-se ressaltar, com esta pesquisa, que a busca por um atendimento de urgência e também por aqueles tratamentos que não são urgência é grande no âmbito educacional do nível superior, de forma a prevalecer o tratamento conservador, contribuindo, dessa maneira, com a saúde bucal, sendo importante priorizar o estabelecimento de um diagnóstico preciso, visando o bem-estar do paciente, bem como a melhora da qualidade do atendimento. Esses resultados permitem avaliar a importância fundamental de um serviço de urgência em uma faculdade para a elaboração de melhores proposições de estratégias no campo da prevenção, assim como a imprescindível formação de profissionais capacitados para o atendimento em situações de urgência odontológica.

\section{Conclusões}

O perfil epidemiológico dos pacientes, atendidos na urgência da FOP/UPE, apresentou uma predominância para o gênero feminino, com a faixa etária mais utilizada no serviço incluída na segunda e na terceira décadas de vida. A dor foi o principal motivo da busca por atendimento nesse setor, sendo a urgência odontológica mais prevalente a de origem endodôntica, com significativa frequência da pulpite irreversível sintomática, e, consequentemente, o tratamento mais realizado nessa clínica foi a pulpectomia, demonstrando que o alto percentual de urgências, com patologias pulpares, é reflexo ainda da presença significante da cárie dentária na população.

\section{Abstract}

Objective: this study aimed to assess the prevalence of dental emergencies, as well as the most common treatments performed in the emergency department of the School of Dentistry at the University of Pernambuco (FOP/UPE). Materials and method: it is a retrospective study based on secondary data, using emergency reports from the period of 2007-2011 as a research instrument. Data were collected using a standard form. The significance level considered in the statistical tests was $5 \%$, and descriptive statistical techniques were used through the development of absolute and percentage frequencies, and the statistical measures: mean, standard deviation, and median. The statistical program used for data analysis was SPSS (Statistical Package for Social Sciences) version 17. Results: 862 reports were obtained from patients of both genders, with a majority of women (70\%), age ranged from 12 to 81 years, and the most frequent complaint was toothache (80.3\%). The most common diagnosis was symptomatic irreversible pulpitis (29.6\%) followed by acute dentoalveolar abscess (7.8\%), and the most frequently performed treatment at this dental service institution was pulpectomy (26.8\%). Conclusions: it may be inferred that pain was the most important reason for seeking dental treatment in the emergency department of the FOP/UPE, reflecting the high percentage of endodontic emergencies in the dental context. Therefore, this study is important for contributing to the development of the epidemiological investigation of dental emergencies in the broad sense of the oral health context of the population.

Keywords: Epidemiology. Emergency. Pain. 


\section{Referências}

1. Sanchez HF, Drumond MM. Atendimento de urgências em uma Faculdade de Odontologia de Minas Gerais: perfil do paciente e resolutividade. ver. Gaúcha Odontol. Porto Alegre 2011; 59(1):79-86.

2. Simas LJP. Glossário odontológico. São Paulo, Pancast 1989; p. 253.

3. Silva CHV, Araújo ACS, Fernandes RSM, Alves KA, Pelinca RN, Dias YC. Perfil do serviço de pronto atendimento odontológico da Universidade Federal de Pernambuco. Odontologia Clín. Científ., Recife 2009; 8(3):229-35.

4. Guimarães MM, Marcos B. Perda de dente relacionada a razões clínicas segundo a classe social. ver. CROMG 1995; 1(2):54-61.

5. Guimarães MM, Marcos B. Impacto da classe social nas extrações de dente. Rev. CROMG 1996; 2(2):78-82.

6. Guimarães MM, Marcos B. Expectativa de perda de dente em diferentes classes sociais. ver. CROMG 1996; 2(1):16-20.

7. Kanegane K, Penha SS, Borsatti MA, Rocha RG. Ansiedade ao tratamento odontológico em atendimento de urgência. Rev. Saúde Pública 2003; 37(6):786-92.

8. Mialhe FL, Possobon RF, Boligon F, Menezes MA. Medo Odontológico entre Pacientes Atendidos em um Serviço de Urgência. Pesq. Bras. Odontoped Clin Integr, João Pessoa 2010; 10(3):483-7.

9. Cohen LA, Manski RJ, Hooper FJ. Does the elimination of medicaid reimbursement affect the frequency of emergency department dental visits? Jada 1996; 127:607-9.

10. Ferreira JO, Damante JH. Serviço de urgência odontológica: aspectos epidemiológicos e administrativos. RPG Rev Pos$\operatorname{grad} 1998 ; 5(1): 31-8$

11. Carnut L, Figueiredo N, Goes PS. Avaliação do nível de satisfação dos usuários das urgências odontológicas da cidade do Recife. UFES Rev. Odontol 2008; 10(3):10-5.

12. Dourado AT, Caldas AF Jr, Albuquerque DS, Sá Rodrigues VM. Estudo Epidemiológico de Urgências Odontológicas. J. Bras Clin Odont Int 2005; 9(48):60-4.

13. Oliveira PC, Zanetta-Barbosa D, Souza HJ, Batista JD, Ranali J, Costa MDMA et al. Avaliação do nível de ansiedade e dor de pacientes em urgências endodônticas e sua influência sobre parâmetros cardiovasculares. Cienc Odontol Bras 2007; 10(4):70-5.

14. Marchini L, Patrocínio MC, Rode SM. Plano de tratamento em uma unidade de urgências e emergências em odontologia. Rev. Fac. Odontol. São José dos Campos 2000; 3(1):85-90.

15. Moura LB, Blasco MAP, Machado HH, Xavier CB. Epidemiologia dos pacientes atendidos no serviço de urgência da unidade de Cirurgia Buco-Maxilo-Facial da FO-UFPEL. In: XIX CIC XII ENPOS II Mostra científica: 2010; Pelotas. Anais. UFPel 2010:1-4.

16. Tortamano IP, Costa CG, Moraes LJ, Borsatti MA, Rocha RG, Tortamano N. As urgências odontológicas e o tratamento clínico e medicamentoso integrado. J Bras Clin Odontol Integr 2004; 8(43):78-85.

17. Leopoldino VD. Perfil dos pacientes que demandam por tratamento no Setor de Urgência da Faculdade de Odontologia da Universidade de São Paulo. [Dissertação de Mestrado]. São Paulo: Faculdade de Odontologia, Universidade de São Paulo; 2004.

18. Galvão MWG, Neves CM, Francisco SS. Levantamento dos atendimentos de urgência do curso de odontologia da Unievangélica. In: $15^{\circ}$ CIOGO - Congresso Internacional de Odontologia de Goiás: 2009; Goiana. Anais. Robrac 2009; 18(46):46.
19. Tortamano IP, Leopoldino VD, Borsatti MA, Penha SS, Buscariolo IA, Costa CG et al. Aspectos epidemiológicos e sociodemográficos do Setor de Urgência da Faculdade de Odontologia da Universidade de São Paulo. Rev. Pós-Grad. 2007; 13(4):299-306.

20. Souza TL, Baptista LCP. Estudo epidemiológico das urgências odontológicas nas USB do Município de Embu das Artes. In: Congresso de Iniciação Científica 11: 2008; Mogi das Cruzis, São Paulo. Anais. Copyright UMC; 2008. p.91-2.

21. Cassal JB, Cardozo DD, Bavaresco CS. Perfil dos usuários de urgência odontológica em uma unidade de atenção primária à saúde. Rev. APS 2011; 14(1):85-92.

22. Matos C. Folha Uol - Brasil têm 96 homens para cada 100 mulheres aponta censo. [Citado 2012 jul 30].Disponível em URL: <http://www1.folha.uol.com.br/cotidiano/908784-brasil-tem-96-homens-para-cada-100-mulheres-aponta-censo $>$.

23. Mascarenhas MDM, Silva MMA, Malta DC, Moura L, Goes PSA, Moysés ST et al. Perfil epidemiológico dos atendimentos de emergência por lesões bucodentais decorrentes de causas externas, Brasil, 2006 e 2007. Cad. Saúde Pública, Rio de Janeiro 2012; 28:124-32.24. Segura JJ, Jiménez RA. Urgências endodônticas: análise epidemiológica de 80 casos. J Endod Pract 2002; 1(2):16-23.

25. Roncalli AG. Projeto SB Brasil 2010 - Pesquisa nacional de saúde bucal revela importante redução da cárie dentária no país. Cad. Saúde Pública 2011; 27(1):4-5.

26. Camargo MBJ, Barros AJD, Frazão P, Matijasevich A, Santos IS, Peres MA. Preditores da realização de consultas odontológicas de rotina e por problema em pré-escolares. Rev. Saúde Pública 2012; 46(1):87-97.

27. Amorim NA, Silva TRC, Santos LM, Tenório MDH, Reis JIL. Urgência em Odontopediatria: Perfil de atendimento da Clínica Integrada Infantil da FOUFAL. Pesq Bras Odontoped Clin Integr 2007; 7(3):23-7.

28. Menini MO, Gomes BPFA, Ferraz CCR, Souza-Filho FJ, Zaia AA. Avaliação do sucesso clínico do atendimento de urgência endodôntica na FOP - Unicamp. Rev. Assoc. Paul Cir Dent 2007: 61(1):39-43.

29. Knackfuss AP, Costenaro RGS, Zanatta FB. Dor odontológica e indicadores de risco em jovens. Rev. Gaúcha Odontologia 2011; 59(2):185-91.

30. Lacerda JT, Simionato EM, Peres KG, Peres MA, Traebert J, Marcenes W. Dor de origem dental como motivo de consulta odontológica em uma população adulta. Rev. Saúde Pública 2004; 38(3):453-8.

31. Carvalho JC, Rebelo MAB, Vettore MV. Dor dental nos últimos 3 meses em adolescentes e estratégia saúde da família: a comparação entre duas áreas com abordagens diferentes de atenção em saúde bucal. Ciência \& Saúde Coletiva 2011; 16(10): 4107-14

32. Munerato MC, Fiamingh DL, Petry PC. Urgência em Odontologia: um estudo retrospectivo. Rev. Fac. Odontol Porto Alegre 2005; 46(1):90-5.

\section{Endereço para correspondência:}

Elivalter Pereira Martins.

Espinheiro, nำ195/802

Ed: Praia das Oliveiras - Bairro Espinheiro

52020-020 Recife - PE

E-mail: elivalterpereira@gmail.com

Recebido: 02/06/2014. Aceito: 03/12/2014. 